\title{
FURTHER RESEARCHES
}

ON THE

\section{PATHOLOGY OF PHLEGMASIA DOLENS.}

\author{
BY \\ ROBERT LEE, M.D., F.R.S., \\ FRLLOW OF the roYal COLlege OF PHYSicians, LONDON; \\ PHYSICIAN TO THE BRITISH LYING-IN hOSPITAL; \\ AND LECTURER ON MIDWIFERY AT SAINT GEORGI'S HOSPITAL. \\ Received April 11th.-Read May 10th, 1853.
}

More than a century and a half had elapsed from the time when the disease termed, l'enflure des jambes et des cuisses de la femme accouchée, depôts laiteux, and phlegmasia dolens, was first described by Mauriceau, and an attempt was made to discover, by morbid anatomy, the proximate cause or the true nature of the affection. Before the publication of the memoirs of M. Bouillaud, the late Dr. Davis, and $M$. Velpeau, various hypotheses had been advanced respecting the cause of the swelling in the lower extremities of puerperal women, but they were mere speculations, unsupported by facts, and inadequate to account for the symptoms. The cases and dissections related by these authors first threw light on the real nature of the disease, and showed that it consisted in an inflammation of the trunks and principal branches of the veins of the lower extremities.

My papers, published in vol. XV of the 'Medico-Chirur- 
gical Transactions,' contained a report of thirteen cases of phlegmasia dolens, in six of which the actual condition of the iliac and femoral veins was ascertained by dissection. From this I was led to infer, that inflammation of the iliac and femoral veins gives rise to all the phenomena of that disease in puerperal women, and "that in phlegmasia dolens the inflammation commences in the uterine branches of the hypogastric veins, and subsequently extends from them into the iliac and femoral trunks of the affected side."

The second of these papers contained the histories and dissections of three cases of cancerous ulceration of the os and cervix uteri, in which there was inflammation of the internal, common and external iliac and femoral veins, and all the characteristic symptoms of puerperal phlegmasia dolens.

Mr. Lawrence, in vol. XVI of the Transactions of this Society, has recorded a similar case, and Mr. Holberton two cases, in which crural phlebitis followed ulceration of the mucous membrane of the intestines.

According to the concurrent experiments performed on dogs by Pirigott, in $1839,{ }^{1}$ and Reumert, in $1840,{ }^{2}$ the tendency to the extension of inflammation in veins otherwise healthy is not so great as is commonly supposed. The action of chemical and mechanical irritants remained for the most part limited to the vein, on which the experiment was made, and extended further only when the vein was at the same time laid bare, or when the foreign body was kept in continued contact with it. Whether inflammation of venous trunks admits of being excited from constitutional causes independently of local irritation, Stanius considers doubtful, after having collated and tested all the facts bearing on the subject. ${ }^{3}$

- Ann. der Chirurg. Abth. des Clinikums von Dorpat Zweiter Jahrg. ; Dorp. 1839.

2 De Symptomatibus Inflammationis Venæ Cavæ Diss. Inaug.; Havn. 1840, p. 46.

${ }^{3}$ Ueber Krankhafte Verschleissung grössen Venenstämme; Berlin, 1839. 
A series of experiments on the lower animals similar to those now described, have recently been made in this country, and a paper on Phlegmasia Dolens has been read to this Society during the present session, not founded on actual observation of the disease, as it occurs in the human subject, but upon these experiments on the veins of the lower animals, in which phlegmasia dolens has never been observed.

The object of the present communication is to submit to the consideration of the Society the observations which I have made during the last twenty-four years on inflammation of the crural veins.

Case xiv.-A lady, æt. 26, was delivered prematurely of her first child, on the 19th of June, 1831. The placenta being retained beyond the usual period was extracted artificially. In a few days, pain in the region of the uterus, with fever, and great prostration of strength, took place. After the application of leeches, the tenderness of the hypogastrium disappeared, but the pulse continued frequent till the end of the third week, when a painful sense of tension was experienced along the brim of the pelvis, on the left side, and this was speedily followed by phlegmasia dolens in the left lower extremity.

On the 21st July, about a month after delivery, I saw the patient with Mr. Cleland, of Rock Hill, Ratcliff. The pulse was 150, and feeble. There was constant nausea, vomiting, and diarrhœa. The tongue was of a dark brown hue. The countenance and whole surface of the body was of a yellowish dusky colour. The respiration was hurried, with frequent cough and expectoration; there was great debility and occasional delirium. The whole of the left lower extremity was swollen to nearly double the size of the other. The femoral vein, exquisitely tender on pressure, felt large and hard in the upper part of the thigh, and there was fulness and tension above Poupart's ligament, in the situation of the iliac veins. The foot and ankle pitted upon pressure; but the integuments of the thigh 
were hot and tense, and did not retain the impression of the finger.

On the 22d, there was great prostration of strength. Pulse 160. Respiration laborious. Tongue dry and brown; diarrhœa, and vomiting. She was conscious at intervals, and then complained of great pain along the inner part of the left thigh, and in the ham. The abdomen was tympanitic.

Death took place on the 24th; and I examined the body with Dr. Sims and Mr. Cleland, on the 25th.

The uterus had sunk down into the pelvis, and was as much reduced in size, as it usually is four weeks after delivery. The peritonæum at first sight appeared every where healthy, but on closer inspection, an adhesion by means of false membrane was found to exist between the posterior part of the uterus and rectum. More than a pint of fluid was contained between the uterus and rectum. The peritonæal and muscular coats of the fundus and body of the uterus were so soft as to be readily torn with the fingers, and of an inky black colour. On laying open the uterus the placenta was found to have adhered to the posterior and inferior part of the uterus; and the branches and trunk of the left internal iliac vein were all filled with purulent fluid, and their inner surface lined with a false membrane of a blackish colour. The coats of the common, external iliac, and femoral veins to the middle of the thigh were all thickened, and their cavities filled with soft coagula of lymph and pus. The vena cava to about two inches below the hepatic veins, was completely blocked up with a coagulum of lymph, which partially adhered to the inner surface of the vessel. Several glands in the vicinity of the vena cava and iliac veins were in a state of suppuration. The coats of the left internal iliac vein at its termination in the common iliac were in a soft shreddy state. The right common, internal and external iliac and femoral veins, were all in a healthy condition.

CASE xv,-On the 27th of January, 1832, a patient of 
the British Lying-in Hospital was delivered after a natural labour. Obscure febrile symptoms soon took place, without any uterine pain, and $I$ was not called to see her till ten days after her confinement, when the pulse was 130 ; the tongue of a dark glossy red colour; the lips parched; the countenance of a dusky yellow colour; tremors of the muscles of the face and extremities, with occasional delirium. There was no pain, tension, or swelling of the hypogastrium; but there was exquisite pain on pressure along the course of the iliac vessels, and down the inner part of the thigh, on the left side, and the whole of the left lower extremity was much swollen, hot, tense, and shining.

On the 13th, the pain in the course of the iliac and femoral vessels had been relieved by leeches and fomentations. The pulse was 140. The tongue dry and brown. The conjunctivæ of both eyes had suddenly become intensely red and swollen, and the vision impaired, if not lost. The right knee-joint had become exquisitely painful when moved; but it was neither red nor swollen. A dark coloured gangrenous spot had appeared over the sacrum, and she died on the 18th.

The uterus had subsided into the pelvis, and there was no trace of disease perceptible in the peritonæum. Both spermatic veins were healthy. The coats of the left common, external iliac, and femoral veins, deep and superficial, were all thickened, and their cavities plugged up with firm coagula. The same was the case with the epigastric vein, and circumflexa ilii. The glands in the vicinity of these veins were enlarged, red, and vascular, and closely adherent to the cellular membrane and outer surface of the vessels. The vena cava, to a short distance above the entrance of the left common iliac vein, had its coats thickened, and a soft coagulum of lymph adhering to its inner surface. The uterine, vaginal, gluteal, and most of the other veins, which form the left internal iliac, were gorged with pus, and lined with false membranes of a dark colour approaching to black. The uterine branches of the right internal vein were also filled with pus and lymph; but the inflammation had not 
extended beyond the entrance of the trunk of the vessel into the common iliac, and the right common external iliac and femoral veins were all in a healthy condition. In the muscular tissue of the cervix uteri on the left side, was a cavity which contained $3^{i i j}$ of purulent fluid. The veins proceeding from this part of the cervix were filled with pus, and the muscular coat of the body of the uterus was as soft as lard. The conjunctivæ, which before death had been red and swollen, were now almost colourless, scarcely a vessel containing red blood being discernible. The deeper-seated parts of the eye were not allowed to be examined.

CASE Xvi.-Several years ago, I was requested to see a young woman in labour, who resided near the Seven Dials, in whom it became necessary, from the presence of a large fibrous tumour in the walls of the uterus, to have recourse to the perforator and crotchet. In a few days, rigors, rapid pulse, and other symptoms of uterine phlebitis, occurred, and subsequently, phlegmasia dolens in the left lower extremity. Death took place, and on examining the body, the fibrous tumour was found in a softened broken-up condition. The left internal iliac vein filled with pus and lined with false membrane, and the coats thickened, and coagula of blood distending the common and external iliac veins.

CASE xvir.-A young healthy woman, a patient of the Southwark Lying-in Institution, was delivered, after a natural labour, on the 21st October, 1832. Rigors, uterine pain, headache, and fever, soon took place.

On the 31st she complained of great tenderness in the situation of the symphysis pubis, and in the right groin, extending along the course of the femoral vessels, which felt hard and cord-like, beneath Poupart's ligament, for a space of three inches down the thigh. The limb greatly swollen and œdematous, was free from discoloration except below the ham, where it was of a dark hue, from distension of the saphena veins. The pulse became very rapid, diarrhœa and gangrenous inflammation of the parts covering the 
sacrum, and the right outer ankle and foot, and death took place soon after. The bones of the pubis were found separated, a quantity of pus in the pelvis, and the right femoral vein plugged up with fibrine, and the saphena major filled with pus. Permission could not be obtained to examine the state of the internal iliac veins.

CASE xviri.-On the 31st December, a young woman died in the St. Martin's Parochial Infirmary, five or six weeks after her confinement. Before pregnancy, she had suffered from cough dyspnœa, and other symptoms of tubercular phthisis. The symptoms became aggravated after delivery, and it was supposed before a post-mortem examination was made, that she had died from chronic disease of the lungs. Before her death she had also suffered from obscure symptoms of uterine inflammation, for which leeches had been applied to the hypogastrium. I examined the body with the late Mr. Gosna, and received from him the report of the symptoms.

The inferior lobe of the lungs on the right side was hepatised and encrusted with lymph. On the left side there were extensive adhesions, not recent. There was no peritonæal inflammation. The uterus had descended into the pelvis, and was of the usual size, four weeks after delivery. The left fallopian tube adhered to the uterus low down behind. The fundus uteri and posterior part of the body were of a peculiar yellow colour. Two patches of a blackish colour were observed near the cervix, where the corpus fimbriatum adhered. There was a small abscess in the left broad ligament. The sinuses in the cervix and body of the uterus contained pus. The branches of the internal iliac veins were all filled with a soft, yellowish coagulum of lymph. This was traced to the common and external iliac, which were both filled with coagula of soft lymph, in the centre of which was a thin matter like pus. About one inch above the entrance of the internal into the common iliac the coagulum ended abruptly, and a dark coagulum of blood, which did not adhere closely to the vessel, occupied 
the greater part of the remainder of the common iliac. A coagulum filled a great part of the vena cava. Cough and scanty viscid sputa, and pain in the chest. She was ordered calomel, and opium, and leeches, and a blister to the chest. Rigors and perspiration came on, also diarrhœa and vomiting, and she sank and died.

Case xIx.-On the 12th October, 1842, I examined the body of a woman who had suffered from phlegmasia dolens in both lower extremities, the left having been first and most severely affected. She had been under the care of Mr. Brookes, of Bedford Street, and Mr. Jones, Soho Square, and before death, she had suffered chiefly from great debility, fever, constant sickness, and diarrhœea. All the pelvic viscera, blood-vessels, and nerves were removed. There was no trace of peritonæal inflammation about the uterus, and the intestines were perfectly sound. All the veins of the cervix uteri on the left side were filled with pus and lymph; and coated with false membranes. The left internal common and external iliac and femoral veins were in the same condition. The coats of the vena cava were greatly thickened as high as the diaphragm, and a rough false membrane adhered to the whole of its inner surface. Within this was a soft yellowish substance, which washed away like a mixture of pus and lymph, with coagula of blood. A quantity of purulent matter escaped from the vena cava when it was opened near its bifurcation; and at this point there was a firm union between the vein and the last lumbar vertebra and cartilages. The right common external and internal iliac and femoral and saphena veins, were all destroyed by inflammation, though less disorganised than on the left side. The left spermatic vein from the uterus to its termination in the renal vein, was thickened and lined with false membranes, and filled with coagula of blood. The cellular tissue of the limb was filled with serum.

CASE xx.-A young lady was delivered of her first child, after a protracted labour, at the end of June, 1844. In the 
course of a few days uterine inflammation took place, which was relieved by antiphlogistic treatment. Six weeks after a second attack of uterine inflammation took place, which was speedily followed by phlegmasia dolens in the left lower extremity; from this she was likewise relieved. In August she had a violent attack of peritonitis, by which she was nearly destroyed. At Ramsgate, where she had gone for the sea air, another attack took place, which reduced her to a state of great debility.

On the 27th October, after a slight irregularity of diet, was seized with violent pain and vomiting, collapse soon took place, and she died on the 30th. The body was examined on the lst November.

Extensive old adhesions were found between the peritonæum and uterus, and quantities of recent lymph. All the veins of the uterus on the left side were filled with coagula. The coats of the internal, common and external iliac veins, and lower part of the vena cava, were all thickened and plugged up. There was nothing unusual observed in the limb.

CaSe XXI.-Mrs. G - was delivered with the forceps on the 10th October, 1844, after having been upwards of fortyeight hours in labour. In a few days fever and dyspnoea took place.

On the 6th November the symptoms were more severe, and the vision of the right eye was suddenly lost.

On the 8th the iris was covered with lymph, and the eye was greatly swollen. There was severe pain in the right shoulder-joint, and in the right hand and fingers, which were stiff and swollen. The whole of the left lower extremity was affected with the swelling, and other symptoms observed in phlegmasia dolens, and there were two large purple spots on the inner part of the left leg; pulse 130.

On the 10th the symptoms were aggravated, and a large gangrenous spot had formed over the sacrum.

She died on the 16th, and I examined the body on the 17th.

XXXvi. 
There was no inflammation of the peritonæum. The left internal iliac vein, through its whole course, was lined with a thin false membrane, which closely adhered to the inner surface of the vessel, and near the uterus its cavity was filled with pus. All the veins on the left side of the os and cervix uteri, and those of the vagina, were inflamed and full of purulent fluid. The coats of the left common iliac were thickened, and its cavity filled with a soft pultaceous mass, the outer surface of which adhered to the vein. A coagulum of blood, three inches in length, was contained in the lower part of the vena cava which had not been inflamed. The left common iliac vein, the superficial and deep femoral, and the saphena veins, were ull filled with firm coagula of blood; but the coats of none of these vessels presented the alterations of structure usually observed after phlebitis. The cellular membrane of the whole of the left lower extremity was filled with serum. ${ }^{1}$

CASE XXII.-This case occurred in St. George's Hospital, and I am indebted to Dr. Wilson for the following details, and permission to place the preparation on the table of the Society. A specimen of fibrinous coagula in the veins, consisting of a portion of the inferior vena cava, the right common iliac veins, and the left, and also with its bifurcation into the internal and external iliac veins. They all, except the right common iliac, contain quantities of coagula of various colours and consistency. The entire vena cava, inferior as high, but not higher than the beginning of the connections with the liver, a portion of which is visible in the preparation, is affected. At the upper part the coagulum was less abundant, firmer and paler, and the lining smooth surface on which it lay was whiter. But in the lower parts, the venous tunics are equally thickened, and the coagula are in greater abundance, giving a stain to the walls of the vein. The various veins were firmly adherent by consolidated areolar tissue, surrounding them to their respective arteries and contiguous structures, and the lym-

' See paper on Ophthalmia in Puerperal Women. 
phatic glands of the iliac, femoral, and lumbar regions were greatly enlarged. The right common iliac vein is laid open, being quite healthy, and without contents. In addition to the products visible in the veins, there also existed a quantity of dirty-looking greyish-red puriform substance, which, owing to its fluidity, has been washed away. Along with this state of things was found also great distension of the lining surface of the spermatic veins, as well within as without the broad ligament; the left hypogastric and uterine veins were in a similar condition, but did not contain any coagula. On examining the reins within the broad ligaments of the uterus, on the left side, there was found a cavity, containing nasty brown puriform fluid, but apparently not connected with any venous trunk. The veins on the right side were healthy. On examining the uterus, the muscular structure was found healthy, but the mucous lining was destroyed, displaying a free roughened surface of an ash-grey colour, and in the right corner of the uterus was a portion of surface to which a placenta had evidently been attached. The mucous membrane of the os uteri and vagina was flaccid and congested, as well also as the uterus. There was also found degeneration of the kidneys and recent pleurisy, with bronchitis and secondary lobular pneumonia in various stages of progress. Some parts having softened and advanced to the formation of purulent matter; others yet presented the light colour and firm consistency, surrounded by dark congested parenchyma peculiar to the early stage. Throughout the body the blood was very fluid, and the structures generally softened.

The subject of this attack was Elizabeth Gould, æt. 22, a patient of Dr. Wilson's, who died July 11th, 1851, one month after a premature confinement, being at the time only six months pregnant. Parturition was succeeded by abdominal pain and constipation, subsequent diarrhœe and rigors, with pain and swelling in the calf of the leg, came on, and this extended up the thigh. The abdominal pain subsided. On admission into the hospital, the tongue was coated, the pulse quiet and feeble, but the abdomen was 
soft and free from pain. The thigh was very tender on pressure, and swelled. There was dyspncea, cough, and scanty viscid sputa, and pain of the chest. She was ordered calomel, and opium, and leeches, and a blister to the chest. Rigors and perspirations came on, also diarrhœa and vomiting, and she sank and died. In this instance it is remarkable that the state of the veins should have begun, as was doubtless the case, in the uterine veins, and yet they themselves should be so slightly affected; and also, that for some cause or other, the iliac and femoral veins on one side are entirely unaffected, the affection having crept upwards high into the inferior vena cava, and low down into the veins of the other side. ${ }^{1}$

From the whole of the facts now adduced, it may be again "inferred that inflammation of the iliac and femoral veins gives rise to all the phenomena of phlegmasia dolens, and that the inflammation commences in the uterine branches of the hypogastric reins, and subsequently extends from them into the iliac and femoral trunks of the affected side."

The following cases furnish additional evidence in favour of this conclusion, though, in consequence of the recovery of the greater number of the patients, an opportunity was not afforded of determining, by dissection, the actual condition of the crural veins.

CaSe xxirr.-Mrs. W-, æet. 26, was delivered, after a protracted labour, of her first child, on the 9th of July, 1830. In less than twenty-four hours great tenderness of the abdomen came on, with nausea, retching, headache, restlessness, and rapid pulse. Dr. Merriman saw her with me, and venesection, leeches, calomel, and opium were freely employed.

On the 14th the pain in the region of the uterus had disappeared, but the pulse was 140 .

On the 16th the pulse was 126, and the gums were affected with the mercury. She complained of pain in the 
left iliac region, and along the femoral vein, increased by pressure, and there was an unusual fulness in this situation.

August 28th, 1830.-During the last month the pulse has never been below 100, and frequently 110 . There have been slight febrile attacks in the evening, sense of coldness followed by profuse perspirations. The pain in the left side of the hypogastrium has never wholly disappeared, and it has been accompanied with uneasiness over the whole abdomen, and distressing flatulence. On examining the left iliac region it is found to be fuller and harder than natural, and sore on pressure. Along the brim of the pelvis, in the situation of the external iliac vein, there is felt a considerable hardness and swelling; this can be distinctly traced to the distance of an inch or two under Poupart's ligament, where it is very painful on pressure. There is also tenderness along the inner surface of the thigh, but the femoral vein cannot be traced down the limb, and there is no swelling of the superficial veins. The limb afterwards became swollen but in a slight degree, and it appeared probable from the symptoms, that the inflammation had not extended beyond the iliac veins. For a very considerable period the thigh remained contracted upon the trunk and could not be extended.

CASE Xxiv.-On the 17th January, 1831, I was called by Mr. Anson to see a puerperal patient residing at 12, Dorset Street, Manchester Square, who was suffering from phlegmasia dolens in the left lower extremity. The whole limb was swollen, tense, hot, and colourless. The femoral vein could not be distinctly felt, but there was exquisite tenderness on pressure in the left iliac fossa, and along the course of the femoral vein to the ham. There was no pitting on pressure in any part of the limb, and the power of moving it was entirely lost. Pulse 120. Tongue foul. Anorexia. The delivery had taken place three months before. It was observed that the pulse continued unusually quick from that time till the appearance of the swelling of 
the limb, which took place on the 16th. The intumescence of the lower extremity was preceded by tenderness of the hypogastrium and left groin, sickness, and remarkable depression of strength and spirits. The symptoms were soon relieved by the repeated application of leeches along the course of the iliac and femoral veins, fomentations, and other means.

Case xxv.—Mary Eggius, æt. 19, No. 20, Little Maryle-bone Street, a patient of the Middlesex Hospital, under the care of Dr. Hugh Ley, was delivered of her first child on the 26th of November, 1831. The labour was naturul, and there was no enlargement of the veins of the lower extremities during pregnancy. On the sixth day after delivery she was attacked with rigors, vomiting, great sensibility of the hypogastrium, and suppression of the lochia. On the ninth day pain was experienced in the left groin, and soon after a swelling took place in the calf of the leg and ham, which gradually extended over the whole limb. For seven weeks the extremity continued swollen to double the size of the other, hot and painful, and incapable of being extended or moved.

January 25th, 1832.-The limb is still larger than the other, and pits. She suffers from occasional severe attacks of pain in the lower part of the abdomen, and along the course of the femoral vessels. The superficial veins of the lower part of the abdomen and upper part of the thigh are enormously enlarged. Around the ankles there are large clusters of varicose veins. Blisters, which produced extensive ulcerations, were applied to the calf of the leg without benefit. The general health is much impaired, and she has only partially recovered the use of the limb.

Case xxvi.-On the $3 d$ December, 1834, I saw a lady, with Mr. Powell, at 66, Grove Street, Camden Town, in whom crural phlebitis on the right side had taken place two months before, subsequent to a natural labour. The attack commenced with the usual symptoms of inflammation of the 
veins of the uterus. When I visited the patient, who had previously been seen by Mr. Stanley, the affection of the limb was subsiding, but there was still tenderness along the course of the femoral vessels, and the femoral vein could be felt hard and large. In the left inferior extremity, all the branches of the saphena vein were dilated around the knee. On the left side there was no tenderness along the course of the iliac and femoral veins; but there was reason to suspect that the inflammation of the right common iliac had extended into the vena cava, and probably into the left common iliac vein.

Case xxvir.-On the 3d December, 1834, Sir Astley Cooper requested me to see the wife of an old and faithful servant, who was dying after delivery at 71, Portland Street, Portland Town, and who had been attended by Mr. Love, of Brook Street. She was delirious, there was rapid feeble pulse, cold extremities, profuse perspiration, and diarrhœa. The whole of the left lower extremity was swollen but not discoloured, and there was great tenderness along the course of the iliac and femoral veins on the left side. The veins around the knee were unusually turgid.

The usual symptoms of suppurative uterine and crural phlebitis were present, and she died on the 18th, after extensive sloughs had formed over the sacrum. The husband would not permit the body to be examined.

Case xxviri. - On the 12th October, 1835, I saw a patient in St. George's Hospital, under the care of Dr. Seymour, who had been delivered in the Westminster Lying-in Hospital, in February. The labour was natural, but she was attacked soon after with uterine inflammation, for which she was copiously bled, and had warm poultices applied to the hypogastrium. She had remained in a weak state during the summer and autumn; but suckled her child. About the beginning of September, headache and febrile symptoms came on, and pain in the right groin. A week after, the right foot and leg began to swell. The 
whole of the right lower extremity is now swollen, hot, and colourless. The foot pits on pressure. Along the leg, thigh, and hip, the superficial veins are greatly dilated. In the right groin, there is a hardness felt, which is painful on pressure. I could not be sure that this was the femoral vein. She had been ruptured on this side. Slight coloured discharge from the vagina. Os uteri healthy. The uterus in its usual situation. Nothing unusual within the pelvis. Pulse 80. Tongue clean. Appetite indifferent. Bowels regular. No cough nor difficulty of breathing, nor pains in the joints.

October 28th.-A few leeches were applied to the right groin, and a tepid lotion with Liq. Ammon. Acetat. to the limb; and the swelling of the limb gradually subsided.

Case xxix.-July 16, 1836, Mrs. D-, æt. 25, Kensington. First child; tedious labour. Now complains of pain and tension in the left iliac fossa, pain on pressure along the inside of the thigh to the knee, then in the ham. Foot and leg swollen, hotter than the other, not pitting on pressure. Not discoloured. Pulse rapid; rigors, sickness at stomach, diarrhœa. Leeches, fomentations, and poultices were applied, and anodynes exhibited internally.

July 17th.-Less pain in the limb, and no increase of swelling. The leeches bled well, and decided relief followed the bleeding.

18th.- Has slept well, and has not complained of acute pain-feels much better. The secretion of milk returning.

25th.-Symptoms declining rapidly. The temperature of the affected limb was ascertained, on the 18th of July, to be $92^{\circ}$, the temperature of the sound limb being $85^{\circ}$. From the 18th to the 24th, the affected limb has been only five degrees higher than the other.

CASE xxx.-21st May, 1840, I saw a lady in whom the usual symptoms of crural phlebitis appeared in a mild form, ten days after delivery. There was pain; first appearance 
in the left side of the hypogastrium, which gradually extended along the course of the femoral vein. The swelling and tenderness slowly subsided, but though twelve years have elapsed since the attack, the circulation is still carried on imperfectly in the limb.

Case xxxi.-On the 24th July, 1840, I saw a lady in consultation, who had been delivered of her first child, on the 7th July, after a tedious labour, complicated with hæmorrhage. On the tenth day after the confinement, she complained of pain like rheumatism in the right lower extremity. The next day there was swelling of the inside of the thigh, and pain along the course of the femoral vessels. Leeches were applied.

22d.-Pain in the ham, the leg uniformly swollen, hot, and tender along the inner surface. No pain along the outside of the limb.

24th.-The temperature of the affected limb higher than the other. The whole limb swollen, tenderness along the brim of the pelvis or the right side, and along the femoral vein. Pulse 100.

August 8th.-The left lower extremity similarly affected. The swelling and tenderness of the right greatly reduced. The affection of the left lower extremity was accompanied with great constitutional disturbance, and the health was seriously injured.

Case xxxir.-In 1841, I saw a lady in consultation, whose first labour was extremely protracted, and delivery was accomplished by artificial means. Great tenderness of the uterine region followed with fever, and in the course of some weeks crural phlebitis took place in both lower extremities in a mild form. Recovery took place; but in 1845, both limbs were still swollen, and the legs and feet covered with varicose veins.

Case xxxirI. - On the 16th June, 1843, with Mr. Cathrow, I saw Mrs. -, æt. 26, in High Street, Mary-le- 
bone, who had been delivered on the 11th September, 1842, of her second child, after a natural labour. Fever took place soon after, with pain in the region of the uterus. Fifteen days after delivery there was pain in the right groin and hip, and the right leg became swollen; and in a few days after the left began to swell, was hot, stiff, and could not be moved. This took place in Northamptonshire, and she has never properly recovered from the attack. Six weeks ago she struck the calf of the leg against a chair, which was followed by pain and swelling. There is now felt a general hardness along the inner surface of the limb, pitting on pressure, the saphena veins are hard and distended, the thigh swollen. Left leg, foot, and thigh hard, and swollen in some parts. Veins distended. No heat of surface. No pain on either side of the hypogastrium. Palpitation of the heart. Tongue clear and moist; appetite good. Catamenia regular. All the means that could be thought of were recommended to restore the free circulation of the blood in the limbs.

Case xxxiv.-On the 19th August, 1843, I saw a patient who had been delivered of her second child, on the 2d February, 1851. The labour was complicated with hæmorrhage, and retained placenta. Symptoms of uterine inflammation occurred in the course of a few days, and this was soon followed by pain in the groins, to which turpentine fomentations were applied. On the tenth day after delivery there were rigors, and the right lower extremity became swollen and painful. Leeches were applied, and mercury given internally. The pain and swelling of the right limb subsided, and the left became similarly affected. Now the left leg has a perfectly natural appearance, the veins are not much distended, and there is no discoloration of the limb; but the patient states that she suffers much from pain about the groins and thighs, and disagreeable nervous sensations about the limb. The right foot and leg are quite well. 
CASE XXXv. - Mrs. D - was delivered of her first child, after a natural labour, on the 4th September, 1843. No symptom of uterine inflammation succeeded, but during the succeeding three weeks she complained of great weakness, feeling of sinking, and the pulse was rapid.

On the 26th September she complained of pain in the left groin and calf of the left leg, the day after there was stiffness and slight swelling, and tenderness in the course of the femoral vein, and all the superficial veins of the limb were distended. Leeches and fomentations were employed.

On the 29th, the swelling had increased, there was great tenderness in the ham, and along the inside of the thigh; and there was slight pitting of the foot on pressure. Pulse 100.

On the 1st of October, Dr. James Johnson saw the case; but though there was still great tenderness along the course of the femoral vein, he could not be made to admit that the iliac and femoral veins were inflamed or obstructed, or that crural phlebitis was the cause of the symptoms usually observed in phlegmasia dolens, œdema lacteum, depôt laiteux dans la cuissé, or any other term applied to the disease.

On the 12th, the affection was declining, but ever since more or less inconvenience has been experienced from the obstructed state of the veins.

Case xxxvi.-Mrs. C- was delivered in Mount Street, of her first child, on the 12th October, 1843. Ergot of rye had been given towards the termination of the labour, but the placenta was removed with difficulty, and hæmorrhage followed its extraction. At the end of the week she had headache, complained of great weakness and of pain in the calf of the right leg and groin, with stiffness of the whole limb. Leeches and fomentations were applied.

On the 11th, the whole limb was much swollen, and sickness at stomach, and diarrhœa came on. The pulse 125 , and feeble. There was great tenderness, not only of the whole hypogastrium, but along the course of the iliac and femoral veins. 
This attack of crural phlebitis was severe, but the patient recovered.

Case xxxvi1. - A lady was delivered in Sussex Square, Brighton, after a natural labour, on the 29th August, 1844. Tenderness in the regions of the uterus, severe rigors, and rapid pulse took place in the course of a few days, and gradually the most marked symptoms of uterine phlebitis followed, viz., rigors and perspirations like those of ague, pains and swellings of the joints of the arms and hands, and in the situation of the right parotid gland, bloody expectoration, and dyspnœa, and before death, which took place on the $3 \mathrm{~d}$ November, phlegmasia dolens in the left lower extremity supervened; and there appeared dark oblong and circular spots on various parts of the body, and extensive sloughing over the sacrum. There were severe nervous symptoms in the progress of the disease.

The body was not allowed to be examined.

Case xxxviri.-On the 30th March, 1844, I saw a lady, with Dr. Scott, at Barnes, who had been delivered of her fifth child ten weeks before. A few days after her confinement she was seized with symptoms of uterine phlebitis. In a short time dyspnca and other symptoms of thoracic inflammation supervened. Three weeks after delivery, the left crural veins became painful, and the whole limb affected with a hot colourless swelling. The fever and pulmonic inflammation continued, and some time after the right lower extremity became similarly affected. The veins of the right leg are now distended, the surface of the limb is of a cream colour, hotter than the other, and does not pit on pressure. There is pain on pressure above Poupart's ligament, on the right side. Sallow complexion. Pulse 120. Left lung dull; respiration short and quick. No expectoration, no sickness, diarrhœa, or rigors.

Blisters were applied to the chest, mild mercurials were exhibited, with anodynes, and slight diet; but death took place not many days after. 
Permission could not be obtained to examine the body.

Case xxxix.-On the 27th January, 1845, Dr. Burnie requested me to see a lady who had been delivered of twins, in De Beauvoir Square, about a month before, and who had not recovered favorably. The pulse was 120 ; there was dyspnœa, with crepitating râle in the chest, and a painful state of the left shoulder-joint with some swelling in the back part of the deltoid muscle. There was no redness, but great tenderness on pressure. There was also great tenderness along the course of the right iliac and femoral veins, and in the situation of the right sacro-sciatic notch. No swelling of the extremity. Pulse rapid and feeble, with diarrhœa.

October 30th.-The whole of the right lower extremity swollen, as in phlegmasia dolens.

The case ultimately terminated fatally.

Case xL.-December 7, 1846, a lady, in Sloane Street, fifteen days after delivery. The affection commenced with pain of the right thigh, iliac nerve, and tenderness of the right groin, and stiffness of the whole limb. Rapid pulse and slight delirium. The whole limb was hot and swollen, and there was slight pitting upon pressure.

The patient recovered.

Case xLI.-On the 11th of February, 1845, I saw a lady at Brompton, who had been delivered three weeks before, without any accident. The veins of the right lower extremity had been varicose during pregnancy, and they had become inflamed soon after the confinement. A chain of abscesses had formed along the course of the right saphena vein, which had been opened, and presented a healthy appearance. A deep abscess had formed in the back part of the left hip, and suppuration was going on in the cellular membrane, over the left trochanter. On the 24th, the pulse was rapid and feeble; there was great pain in the right arm, about the elbow-joint, and a large gangrenous slough 
had formed over the sacrum, without any of the internal organs having become affected. This patient ultimately died completely exhausted.

The following cases demonstrate that phlegmasia dolens may occur wholly unconnected with pregnancy and parturition, and that in such cases the inflammation likewise commences in the uterine branches of the hypogastric veins, and subsequently extends from thence into the iliac and femoral trunks of the affected side. In some of these cases the inflammation of the uterine veins was produced by cancerous disease of the os and cervix uteri, in others there was no organic disease existing previously of any kind.

Case xhir.-On the 9th May, 1835, I saw a young lady in Bruton Street with Mr. Nicholles, who had been seized soon after her arrival from the Isle of Wight with symptoms of low typhoid fever. For some time before this attack commenced she had been chlorotic, had menstruated sparingly, and had suffered from pain in the back and left hip. Some days before I saw her the whole left inferior extremity had become swollen, tense, and hot, but not very painful. It then looked like alabaster. There was considerable tenderness on pressure along the course of the femoral vein, but the vessel itself could not be distinctly felt. The abdomen was soft and nowhere painful on pressure. The pulse was rapid, the superficial veins distended with blood, the tongue dry and brown.

On the 2lst death took place with vomiting and the usual symptoms of low typhus.

On the 22d I was present at the examination of the body. The left common and external and internal iliac veins were thickened, lined with false membranes, and plugged up with coagula of lymph and blood. The internal iliac was most inflamed, and its vaginal and uterine branches contained pus.

Case XLIII.-At the beginning of April, 1836, I saw a lady under the care of Dr. Duffin, who had a fibrous polypus 
the size of a large pear, with a thick root encircled by the os uteri. Little difficulty was experienced in applying a ligature with the double canula; and the tumour came away five days after in a state of slight decomposition.

On the 26th there were symptoms of inflammation about the left uterine appendages, and tenderness along the crural vessels on the same side. The whole left lower extremity became swollen, as in cases of crural phlebitis in the puerperal state, and the disease after running the usual course, in a mild form, terminated favorably.

CASE XLIV.- In the month of May, 1831, I saw a woman, æt. 22, in the Middlesex Hospital, who was under the care of Sir Charles Bell. The superficial veins of the left lower extremity were greatly distended, and the thigh, leg, and ankle were swollen and odematous; there was great tenderness on pressure along the brim of the pelvis, and in the course of the femoral and saphena veins. The right inferior extremity was in the natural state. About six months before, she had experienced a fall in which the lower part of the spine had violently struck the ground; since that time there had been constant pain in the back, with irregular menstruation and prolapsus uteri. Soon after the accident, she also began to suffer from pain along the brim of the pelvis on the left side, extending downwards under Poupart's ligament along the thigh, in the course of the femoral vessels. The veins of the lower extremity in a short time began to enlarge, and also the whole limb became swollen, painful, and odematous.

CaSe XLV. - In the autumn of 1831, I saw a young lady in consultation with Mr. Jones, Soho Square, who was suffering from an attack of crural phlebitis of the left side. The whole left inferior extremity was swollen, tense, hot, and painful, but not discoloured. The femoral vein under Poupart's ligament was felt like a large hard cord, and pressure over it and along the course of the iliac veins of the same side produced great suffering. The limb was completely 
deprived of the power of moving. The thigh did not pit upon pressure, but the integuments of the leg retained the impression of the finger. The tongue was white, the pulse rapid, and there was great irritability of stomach and depression of strength. This attack was referred to the sudden suppression of the catamenia, from the application of cold and wet, which was followed by great uterine tenderness. The acute symptoms were soon subdued by leeches and warm fomentations to the limb, but the extremity remained weak for some months after.

Case xuvi. - In September, 1830, I saw a lady, æt. 40, under the care of Dr. Copland, who, after being exposed to cold, had a violent attack of inflammation of the bowels, for which copious venesection was required. Great tenderness in the hypogastric region, with pyrexia, continued for some time after, when she began to suffer from pain in the situation of the left crural veins. The whole lower extremity became affected with a hot, tense, and painful swelling, as in puerperal crural phlebitis. The affection had scarcely begun to subside in the left extremity, when she began to experience pain and tension above Poupart's ligament on the right side, and the right thigh and leg also became affected with a hot, painful, colourless intumescence. I saw this lady in consultation with Dr. Copland, when the right extremity had become affected, and the disease did not differ in any respect from the crural phlebitis of lying-in women. By the repeated application of leeches, fomentations, \&c., the acute symptoms were soon subdued, but the feet and ankles long remained weak and œedematous.

CASE XLVIr.-A young woman under the care of Dr. Watson, in 1832, died in the Middlesex Hospital from tubercular disease of the lungs. A swelling of the left lower extremity, similar in all respects to crural phlebitis, had taken place some time before death. The uterus was found to be diseased on the left side. The left common iliac and femoral veins had been inflamed, and the left 
internal iliac was converted into a ligamentous cord. It was evident that the inflammation of the veins had originated in the uterus.

CaSe xuviri.-On the 18th May, 1832, I saw in the Middlesex Hospital a young unmarried woman with varicose veins and extensive swelling of the left lower extremity. The uterine functions had been performed irregularly for many months. There was also great tenderness on pressure in the course of the iliac and femoral veins to the middle of the thigh, and little doubt could be entertained that these veins were inflamed and obstructed.

CASE XLIX.-On the 31 st July, 1833, I saw a woman in the Middlesex Hospital, æt. 39, who had been afflicted for more than a year with cancerous disease of the os and cervix uteri. About the 20th of July she had begun to suffer from great tenderness of the hypogastrium, back and hips, and a few days after acute pain took place in the course of the left femoral vein, which was soon followed by a colourless swelling of the whole limb. There was slight pitting on pressure over the tibia, the superficial veins were greatly distended, the femoral vein was hard and painful about four inches in the upper part of the thigh, the pulse was 130 and small.

Case L. - On the 25th of March, 1830, I examined the body of a woman, æt. 50, who had died of carcinoma uteri, in whom four weeks previous to her decease, the usual symptoms of phlegmasia dolens had appeared in the left lower extremity. There was great tenderness in the course of the femoral vein, and along the inner surface of the thigh and leg to the ankle, and the whole extremity had become tense and swollen. The temperature of the surface was increased, but there was no unusual redness of the skin, and pitting on pressure could only be produced around the ankle, and on the upper surface of the foot.

XXXVI. 
The upper part of the vagina, os, and cervix uteri were destroyed by cancerous ulceration, and a large opening formed between the vagina and rectum.

The trunk and branches of both internal iliac veins were partially inclosed in masses of indurated cellular and adipose substances, and inflamed through their whole extent. On the right side the inflammation terminated abruptly at the junction of the internal with the common iliac vein. The right common and external iliac veins were healthy.

The left common and external iliac and femoral veins were all plugged up with firm coagula, and lined with false membranes. The vena cava, from the junction of the common iliac veins to the entrance of the vena cava hepatica, was occupied and distended with a soft coagulum, which at the upper part had the appearance of a clot of blood, and did not adhere to the lining membrane of the vein. The inferior half of the vena cava was filled with a firm coagulum of lymph; which closely adhered to the vessel. This coagulum, which was soft and pultaceous in the centre, was continuous with that in the left common iliac vein.

Case LI. - On the 15th December, 1830, with Dr. Hugh Ley, I examined the body of Mrs. King, æt. forty.-six, who resided at No. 49, Bell-street, Paddington. For upwards of two years she had suffered from great pain in the uterus, sacrum, loins, and thighs, and there had been a copious discharge from the vagina of a serous, purulent, and sanguineous fluid. Her complexion was sallow, and there were frequent attacks of sickness and vomiting. For some time before death, great pain had been experienced when the contents of the rectum and bladder were passing. It was ascertained at an early period, that there was a great, irregular, fungaid mass growing from the orifice of the uterus. In the month of October, 1830, a swelling took place in the calf of the right leg and ankle, which pitted on pressure. The integuments were hot, but not discoloured. On examining the upper part of the thigh, the femoral vein was felt hard, and it was painful on pressure, and there was 
also great tenderness in the course of the external iliac vein. The swelling of the limb continued three weeks, and then gradually disappeared. A month after, the left lower extremity became similarly affected, but in a much slighter degree. In both the swelling had entirely disappeared before death, which took place on the 13th December, 1830 . Two days after, I inspected the body, and found the fundus uteri natural, but the greater part of the body of the uterus had been converted into a thick mass of a caseous consistence. All traces of the natural structure and the orifice and cervix had disappeared, and they had been changed into a substance like lard. Some portions of this mass were as soft as brain. The right common, internal and external iliac and femoral veins were all impervious, their coats thickened, and filled up with firm coagula of blood. The lower part of the vena cava was lined with a false membrane, which adhered to the inner surface of the vessel. A number of large indurated glands surrounded the lower part of the vena cava, right common external and internal iliac veins. In the centre of some of these glands there was a soft substance like lard, or thick cream.

Cases in which crural phlebitis followed inflammation of the saphena veins, and of the deep veins of the lower extremities, from fracture of the tibia and fibula, and the pressure of encephaloid tumours on the thoracic viscera.

Case LII.-On the 8th February, 1836, I saw a woman, æt. 27, who had been cook in a family for a number of years, and had varicose veins of both lower extremities, and eczema over the legs. The surface was moistened, and nitrate of silver applied, which produced great pain. Severe inflammation of the shin followed, and inflammation of the left saphena vein speedily supervened, and this vessel became hard and painful, as far as Poupart's ligament. In the right lower extremity, the same effect has resulted from the application of the nitrate of silver. Leeches and poultices are now applied to the inflamed veins. The pulse is rapid, the 
countenance pale, but there is no other constitutional symptom of phlebitis.

CASE LIIr.-Inflammation of the vena cava, iliac and femoral veins, from fracture of the tibia and fibula. A specimen of fibrinous coagula in the veins in connection with diffuse inflammation of the areolar tissue arising from a compound fracture of the right tibia and fibula, and with large sloughy sores of the integuments of the buttocks, and that covering the trochanters and other prominent parts. The inferior vena cava, to a point about two inches from the liver, and the right common as well as external and internal iliac veins, are seen with some of their smaller branches. They all contained quantities of toughish fibrinous deposits, pretty firmly adherent to the lining surface of the veins, are greatly thickened, and in places the contained deposit is most intimately united to them. The right external iliac and femoral veins contained a mass of a dark rusty colour, and more easily to be detached from the lining surfaces, which were stained. The vena profunda femoris is seen quite void of any deposit. The light-coloured and somewhat rounded portions of tissue seen united to the lower parts of the preparation, are of thickened and condensed areolar tissue, inseparable from the small venous branches in the cavity of the pelvis. Some of these contained small collections of a puriform fluid. In addition to this firmer deposit, the veins contained quantities of dirty, viscid, chocolatecoloured substance, like softened and broken-down coagula. There was also found extensive peritonitis. The thickened hard membrane lining the pelvic organs binding them down firmly, and the areolar and adipose tissue about the lower part of the bladder and rectum contained numbers of small cavities, with consistent purulent matter within them. This was also the case with the vesical and hæmorrhoidal veins. Secondary lobular pneumonia existed also, and in connection with the fracture of the tibia and fibula there were extensive sloughy sinuses.

The subject of this affection was Edward Miles, a patient 
of Mr. Tatum's, who was brought into St. George's Hospital with the above-mentioned fracture of the leg. The wound became unhealthy, and pain in the groin, followed by rigors, came on, and subsequently diffuse inflammation of the areolar tissue of the entire limb, and a gangrenous state of the foot. Bed-sores, and repeated attacks of the inflammation came on, and he sank and died July 11, 1851.

Case LIv.- Inflammation and obstruction of the lower portion of the vena cava, and the right iliac and femoral veins, produced by an encephaloid tumour at the upper part of the chest.

The right lower extremity was enlarged throughout, the superficial veins were distended. There was a tumour of tolerable firmness, the size of an orange, at the upper part of the chest and a little to the right of the upper bone of the sternum. There was also a large tumour, of about the size of two fists, to the right of, and under the right mamma. In both cases the integuments were entirely healthy. The large tumour pressed into the thoracic cavity, united by adhesions intimately with the lungs and pectoral muscles. These tumours presented the same characters, and were examples of encephaloid cancer. The lungs were healthy. The contents of the abdomen were healthy, except the lower part of the vena cava and the right iliac and femoral veins, with their branches, which quantities of thick coagula firmly adherent to the parietes, and going on in parts to softening and conversion into a brownish thin rusty fluid.

This man was 60 years of age. His name was James Lang, and he was under the care of Dr. Wilson, in St. George's Hospital.

Case Lv.-On the 28th September, 1848, I saw a patient, in the Royal Mews, Pimlico, with Mr. Du Pasquier, who died from inflammation of the saphena veins of the right leg, who had been delivered, a few days before, of her first child after a tedious labour. The veins of both lower extremities had been varicose during pregnancy, especially the right. Two days after delivery, she complained of 
stiffness of the left leg above the ankle-joint. The part above the ankle on the inner surface was red and swollen, and the branches of the saphena vein up to the ham hard and painful. The pulse was 130. On the following day she was very restless ; there was great swelling of the leg, and great tenderness along the course of the saphena vein in the thigh (pulse 140) as high as the groin; a few leeches were applied; much irritation; there was sickness at stomach; great prostration of strength; tongue furred. Died the same night.

The body was examined by Mr. Du Pasquier, and the following is his report of the appearances:- " I examined the veins of Mrs. G-'s leg last evening, it was filled the whole length, from the ankle to the groin, with dark coagulated blood, which stained the cellular tissue which surrounded it, with the same colour. There was no appearance whatever of pus either in the vein itself or in the cellular tissue, at least I could not detect it."

The elbow-joint was swollen and puffy, and the veins around it of a dark colour as in the leg.

CASe Lvi.-Mrs. N-, æt. 37, 1st of July, 1830. Three months ago a small ulcer appeared above the left internal malleolus, with much inflammation of the surrounding integuments. A varicose state of the veins of the leg had existed some time before. The ulcerations were healed in three weeks, but the saphena veins along the inner surface of the leg, knee, and thigh to the groin, became hard, and exquisitely painful. This painful condition of the veins has been gradually increasing, and a general hot and colourless intumescence of the whole limb has taken place. The veins around the ankle can now be felt, indurated and knotted, and in three joints along the front of the tibia, there is a circumscribed hardness, with intense redness of the integuments. There is exquisite pain on pressure along the whole course of the saphena vein in the thigh. The femoral vein, three or four inches under Poupart's ligament, is hard and painful, and pain is experienced on pressure 
along the brim of the pelvis. The hypogastrium, more particularly on the left side, is tense and swollen, and she complains of a distressing sense of pulsation or throbbing in the lower part of the abdomen. For several days there has been retention of urine; the countenance is anxious and depressed; there are tremors of the muscles of the face and extremities; tongue furred; occasional retching; urgent thirst; respiration hurried; slight cough ; pulse 120.

4th.-The limb is less swollen, but there is still great tenderness in the left side of the hypogastrium, and along the inner surface of the limb. Constitutional symptoms somewhat relieved. Great prostration of strength.

27th.-Leeches, \&c., have been repeatedly applied along the course of the affected vessels, and the tenderness is now much relieved. Sickness, with foul tongue, and quick pulse; continue.

14th of May, 1830.-Health improved. There is considerable enlargement of the affected extremity, and there are large clusters of purple veins around the ankle. There is now a hard tumour of considerable size in the situation of the left ovarium, and she has lately suffered much from prolapsus uteri, and uterine irritation, with leucorrhœa; she menstruates regularly; the right lower extremity natural.

18th of October, 1831.-There is much hardness and tenderness on pressure in the situation of the left femoral vein. The extremity is still swollen, of a deep purple colour, and the foot and ankle covered with enlarged veins. The abdomen is swollen, but no fluctuation is perceptible.

The cases and dissections of the distinguished pathologists previously referred to, and those contained in this and my other communications to the Society on Phlegmasia Dolens, prove in the most conclusive manner that inflammation of the iliac and femoral veins, is the proximate cause of the disease; and that in puerperal women the inflammation commences in the uterine branches of the hypogastric veins. It has likewise been demonstrated by morbid anatomy, that phlegmasia dolens is a diseasc which may take place in 
women who have never been pregnant, and in the male sex, and that under all circumstances the proximate cause is the same. Respecting the treatment of phlegmasia dolens, considerable diversity of opinion has prevailed. Mauriceau, who first described the disease, was aware of its danger when the swelling of the limb was very great and painful, accompanied with symptoms of inflammation; suppression of the lochia; fever; difficulty of respiration; and great tension of the hypogastrium; but for such cases he has not described any method of treatment. In the milder forms of the disease he chiefly trusted to diuretics and purgatives, and has made no allusion to bloodletting or local treatment.

Puzos, who published three memoirs, 'Sur les Depots Laiteux, appellés communément Lait Repandu,' in 1759, and who described not merely the local but the constitutional symptoms of uterine and crural phlebitis with the greatest accuracy, employed repeated bloodletting from the arm for the hypothetical purpose of evacuating from the blood the milk formed in it during pregnancy and in the puerperal state, and which imaginary milk, he says, "roule confusément avec le sang, dans toute l'habitude du corps." Purgatives, diaphoretics, and hot fomentations, cataplasms, and frictions with volatile liniments, were the remedies he chiefly employed after the repeated abstraction of blood from the arm. Although Puzos was convinced of the necessity of venesection in those depots, accompanied with fever and which at the commencement were acute and inflammatory diseases, he acknowledges that the remedy was not infallible, and that he had lost several patients, "malgrè toutes les saigneés que j'avois pû̀ faire."

"In every case, however," says Dr. Davis, "where I have been a party to the adoption of such practice, or where I have had the opportunity of observing it in the practice of others, I feel it my duty to state that it has completely disappointed expectation." Dr. Davis recommended the local abstraction of blood by leeches, blisters, evaporating lotions, free and constant exposure to the action of the atmosphere, and the internal exhibition of digitalis and blue pill. 
In all the cases of phlegmasia dolens which $I$ have witnessed, the pulse has been so rapid and feeble, and there has been so much constitutional debility, as usually observed in uterine phlebitis, that $I$ have not ventured to recommend general bloodletting. There are cases, however, which have been observed by others, in which the abstraction of a moderate quantity of blood from the arm has appeared at once to break the force of the attack. In a great proportion of cases venesection is not required, and we may trust for the relief of the inflammation to the repeated application of leeches above and below Poupart's ligament in the course of the iliac and femoral veins. Two dozen of leeches should be applied immediately after the commencement of the disease, and the bleeding should be encouraged by warm fomentations and warm linseed meal poultices. Should the relief of the pain not be considerable, it is requisite to reapply the leeches in numbers proportioned to the severity of the attack, and to repeat them a third, or even a fourth time, at no very distant intervals, should the disease not yield.

Some patients have derived the greatest relief from the use of warm cataplasms to the limb, while others have derived most advantage from tepid evaporating lotions.

The bowels are often much disordered in this disease, but the employment of acrid cathartics is injurious. Repeated small doses of calomel and antimonial powder should be given, with some mild purgatives, not only with the view of correcting the deranged state of the digestive organs, but to subdue the local inflammation and the great constitutional disturbance usually present. It is of importance also to administer saline and diaphoretic medicines, and to procure rest, and relief from pain by anodynes, until the acute symptoms pass away. The diet should be the same as that usually allowed to patients who are labouring under inflammatory and febrile diseases. I have seen no advantage derived from the use of digitalis in any stage, either of uterine or crural phlebitis.

When the acute inflammatory symptoms have passed 
away, the limb remains in a weak and œdematous state, and great uneasiness is often experienced from congestion of blood in the veins. Until the collateral branches which are to carry back the blood to the head become enlarged, it is impossible by any means to afford complete relief. Much benefit may, however, be derived in this stage of the complaint from the application of leeches to different parts of the limb, where there is much tenderness, and by piercing it in the horizontal position. I have seen mischief produced by having recourse too early to remedies intended to promote the absorption of the fluid effused into the cellular membrane. Blisters, friction, stimulants, embrocations, and bandages to the limb, are only useful when the inflammation of the veins has subsided, and other vessels have become so much enlarged as to carry on the circulation of the blood in the extremity without interruption.

I have not perceived any sensible benefit accrue from the use of mercurial ointment and iodine in phlegmasia dolens, and I consider the local abstraction of blood at the commencement of the disease to constitute the most important part of the treatment.

\section{Postscript ; September, 1853.}

Case xxxv.- This lady died from tubercular phthisis, on the 16th Sept. 1853, and on the 18th George Pollock, Esq. examined the body. He removed the iliac and femoral veins of the left side, and they are now in my possession. Bands of false membrane are seen extending across both the iliac and femoral veins, and their canal is greatly contracted but not wholly obliterated. All the structures surrounding the veins appeared healthy. 